\title{
The COVID-19 reset: lessons from the pandemic on Burnout and the Practice of Surgery
}

\author{
John Romanelli ${ }^{1}$. Denise Gee ${ }^{2}$ John D. Mellinger ${ }^{3}$. Adnan Alseidi ${ }^{4}$ James G. Bittner ${ }^{5}$. Edward Auyang ${ }^{6}$. \\ Horacio Asbun ${ }^{7} \cdot$ Liane S. Feldman $^{8}$ on behalf of SAGES Reimagining the Practice of Surgery Task Force
}

Received: 18 August 2020 / Accepted: 30 September 2020 / Published online: 13 October 2020

(c) Springer Science+Business Media, LLC, part of Springer Nature 2020

\begin{abstract}
Background Burnout among physicians is an increasing concern, and surgeons are not immune to this threat. The ongoing COVID-19 pandemic has caused dramatic changes to surgeon workflow, often leading to redeployment to other clinical areas, slowdown and shutdown of elective surgery practices, and an uncertain future of surgical practice in the post-pandemic setting. Paradoxically, for many surgeons who had to prepare for but not immediately care for a major surge, the crisis did allow for reflective opportunities and a resetting of priorities that could serve to mitigate chronic patterns contributory to Burnout. Methods SAGES Reimagining the Practice of Surgery task force convened a webinar to discuss lessons learned from the COVID pandemic that may address burnout.

Results Burnout is multifactorial and may vary in cause among different generation/experience groups. Those that report burnout symptoms often complain of lacking purpose or meaning in their work. Although many mechanisms to address Burnout are from a defensive standpoint—including coping mechanisms, problem solving, and identification of a physician having wellness difficulties—offensive mechanisms such as pursuing purpose and meaning and finding joy in one's work can serve as reset points that promote thriving and fulfillment. Understanding what motivates physicians will help physician leaders to develop and sustain effective teams. Reinvigorating the surgical workforce around themes of meaning and joy in the service rendered via our surgical skills may diminish Burnout through generative and aspirational strategies, as opposed to merely reactive ones. Fostering an educational environment free of discriminatory or demeaning behavior may produce a new workforce conducive to enhanced and resilient wellbeing at the start of careers.

Conclusion Surgeon wellness and self-care must be considered an important factor in the future of all healthcare delivery systems, a need reaffirmed by the COVID-19 pandemic.
\end{abstract}

Keywords Surgeon burnout · Wellness · COVID-19

Prior to the COVID-19 pandemic, concerns about surgeon burnout were steadily growing to crisis levels. Many were

Horacio Asbun and Liane S. Feldman should be considered cosenior authors.

Liane S. Feldman

liane.feldman@mcgill.ca

1 University of Massachusetts Medical School, Baystate Medical Center, Springfield, MA, USA

2 Massachusetts General Hospital, Boston, MA, USA

3 Southern Illinois University School of Medicine, Springfield, IL, USA

4 San Francisco School of Medicine, University of California, San Francisco, CA, USA becoming increasingly alienated from what drew us to surgery and medicine in the first place as we saw an increasing gap between what was important to us professionally and personally and the way the healthcare system required us

5 University of Connecticut School of Medicine and Quinnipiac University Frank H Netter MD School of Medicine, Saint Francis Hospital, Hartford, CT, USA

6 University of New Mexico School of Medicine, Albuquerque, NM, USA

7 Miami Cancer Institute, Baptist Health South Florida, Miami, FL, USA

8 McGill University Health Centre, 1650 Cedar Avenue D6-156, Montreal, QC H3G 1A4, Canada 
to practice. Many became disconnected from the purpose and meaning derived from being able to care for patients, continually improving techniques, belonging to a team, teaching the next generation and feeling appreciated by our colleagues and communities for our contributions. Some of us were just trying to survive, let alone thrive, both at work and in life. Strategies such as mindfulness training, exercise, taking time-off and ways to improve work/life integration seemed to say that the problem was with the doctors, failing to target significant underlying problems related to the electronic medical record (EMR), performance metrics and lack of flexibility and autonomy that diverted us from activities to improve patient care while taking care of ourselves [1].

With concerns about surgeon health and happiness, the Society of Gastrointestinal and Endoscopic Surgeons (SAGES) President Dr. Horacio Asbun convened a group tasked with "Reimagining the practice of surgery". The objectives were to identify strategies that promote joy in the practice of surgery, both through identification of surgeon habits that support optimal performance but also through encouraging institutions to commit to healthier, more efficient and positive work environments. Could surgical practice be redesigned to allow us to reconnect with what motivated us to go into surgery in the first place?

And then, the COVID-19 crisis resulted in a complete reset at work and life. Elective surgery ground to a halt and while many surgeons were re-deployed on teams providing front-line treatment, many others had significantly curtailed elective practices and provided only acute care surgery support. The losses have been enormous and ongoing, and the personal and economic impacts will be felt for years. The lack of knowledge and information, safety concerns at work and at home, worry about the impact on surgical patients, shutting down of research programs and financial repercussions are all substantial. But we also noticed some potentially positive lessons of the pandemic. Within our institutions, we were all pulling together with a singular purpose and collegiality. We found common cause with other healthcare workers providing essential services and our work was recognized and appreciated by our communities. We may have been stressed and tired, but did not question the importance of the work.

For many of us, the stay at home orders had other side effects: we were spending a lot of time with family, working less, using telehealth for patient interactions and online platforms for teaching and meetings, and could not travel. We were more rested, exercising more, and rediscovering non-surgical interests. Although this is not meant to minimize the ongoing impact of the crisis, it is cause for reflection on which of these lessons and opportunities we can potentially use post-pandemic in how to reimagine the practice of surgery.
This manuscript is a summary of a SAGES webinar presentation from May 27, 2020 reflecting on these issues. It begins with a discussion of the challenges in wellness and burnout pre- and post-pandemic, then provides insights for rediscovering meaning and even joy in surgical practice.

\section{Understanding and recognizing wellness and Burnout}

Surgeon wellness is of significant concern and may impact the future health of the profession. Burnout (or moral injury), a term used in medical literature, hospital lexicon, and societal documents, is more prevalent in the surgical community and impactful to surgeons and patients than one might be aware. A surgical community with declining health and wellbeing threatens the provision of safe, high-quality, and patient-centered care. Surgeon wellness is described as "a multi-dimensional commitment that encompasses occupational, mental, physical, emotional, and social domains" [2]. According to Maslach and colleagues, the most commonly cited definition of Burnout is the "triad of emotional exhaustion (overextension), depersonalization (negative, callous, and detached responses to others), and reduced personal accomplishment (feelings of competence and achievement in one's work) [3]. Expanding on the definition by Maslach et al., Burnout refers not only to a decline in wellness but more precisely the culmination of failed wellness and mental wellbeing. Factors associated with Burnout include loss of professional control, autonomy, and flexibility; inefficient processes; disjointed workplace relationships and goals; excessive administrative burdens; frustrations with medical record systems, and poor work-life balance [2].

Given the prevalence and importance of surgeon burnout, it makes sense to investigate if and how the COVID-19 pandemic impacted surgeon wellness. A pre-COVID survey of 15,181 respondents who practice in one of 29 specialties in the USA revealed that $42 \%$ had symptoms of Burnout, down $4 \%$ from 2015 [4]. Results stratified by physician generation showed that almost half (48\%) of respondents identifying as Generation X (born 1965-1980) reported Burnout compared to 38\% of Millennials (born 1981-1996) and 39\% of Baby Boomers (born 1943-1964). Of note, 35\% of general surgeons reported feeling burned out [4]. These findings suggest a disturbing trend away from wellness and mental wellbeing - a trend that may result in the premature loss of talented physicians and surgeons.

Various aspects of healthcare delivery can contribute positively or negatively to surgeon wellness. One contributing frustration among physicians and surgeons is the time spent performing administrative tasks, specifically those related to use (or misuse) of the EMR. The surgeon and author Atul Gawande made valid comments about EMR systems and 
their impact on physician and surgeon wellness in his essay published in The New Yorker. In his essay, "Why Doctors Hate Their Computers," Gawande highlights that "a system that promised to increase my mastery over my work has, instead, increased my work's mastery over me. I'm not the only one.... physicians devoted half of their patient time facing the screen to do electronic tasks. Moreover, these tasks were spilling over after hours.... The result has been epidemic levels of Burnout among clinicians" [5]. The voice of Dr. Gawande is poignant for sure, but his voice is not the only one calling attention to the impact of EMR on wellness and mental wellbeing. Survey data suggest that frustrations associated with EMR use represent the fourth most impactful stressor leading to self-reported physician burnout [4]. Other causes of Burnout among physicians included excessive administrative tasks (55\%), excessive work hours (33\%), lack of respect (32\%), inadequate compensation (29\%), and lack of control or autonomy (24\%) [4].

Another survey including only surgeon respondents listed factors that may be associated with burnout [6]. In this study, $40 \%$ of responding surgeons were burned out. Personal factors associated with increased Burnout included younger age and having a spouse employed as non-physician healthcare professional; having children was protective, but among those with children, those with younger children were at higher risk than those with older children. Professional characteristics associated with higher Burnout were incentive compensation based purely on billing, area of specialization, increased on-call responsibilities, and more hours worked per week. In addition, compensated time ( $>50 \%$ of total work time) for administrative tasks and research seemed to be protective [6].

Factors that may contribute to or protect against Burnout are important to appreciate, but identifying the problem is just the first step toward reclaiming wellness and mental wellbeing. Various coping strategies are critical components of this effort to reclaim and/or maintain wellness. A preCOVID survey of physicians found that $45 \%$ of respondents chose social isolation or exercise as means to cope with negative feelings associated with their doctor role [4]. Others felt that talking with family and friends (42\%), sleeping more (40\%), and playing or listening to music (32\%) helped them stave off symptoms of Burnout. While positive coping strategies may be of significant benefit, others have a negative influence on feelings and behavior. Physician respondents claimed to eat junk food (33\%), consume alcohol (24\%), binge eat (20\%), or use tobacco, marijuana, and/or other drugs (3\%) to combat feelings of burnout [4].

Wellness and mental wellbeing are not issues limited to attending physicians and surgeons. Stressors in medical school and residency training may contribute to Burnout as well. A recent survey of 3,588 postgraduate year 2 (PGY-2) residents across all specialties revealed that $45 \%$ of respondents reported Burnout, 14\% expressed career choice regret, and $14 \%$ would "definitely not" or "probably not" choose to become a physician again. General Surgery was one of five specialties associated with a higher rate of burnout symptoms among PGY-2 residents [7]. Another recent survey of surgical residents reported that $69 \%$ of respondents tested positive for Burnout on at least one subscale [8]. These data strongly suggest the need for further reform in surgical education, and for faster identification of at-risk learners sliding toward the negative end of the wellness spectrum. Initial efforts at reform are evident among surgical residency programs, but the ultimate effectiveness of these initiatives is as yet unknown.

Given the prevalence of impaired wellness and even Burnout among physicians and surgeons at all experience levels, the COVID-19 pandemic, particularly in the hard-hit areas of North America, seriously threatens mental wellbeing. Health systems and surgeons alike face new hardships such as government restrictions on patient care, financial losses, ill-equipped staff, administrative burden, struggle to identify best practices related to COVID-19, and so many more. These new challenges compound an already distressing problem and may push physicians and surgeons already suffering impaired wellness toward full-fledged Burnout.

SAGES examined concerns about surgeon wellness during the pandemic [9]. SAGES concluded that the COVID19 pandemic is taking a significant emotional toll which is exacerbated by fears about personal and family safety, transmission of the COVID-19 virus, clinical redeployment, financial hardship, and reestablishment of a new normal in clinical practice. Moreover, the jarring changes in the conduct of a typical day let alone the practice of surgery may lead vulnerable persons to social isolation [9].

However, there is reason to be optimistic as the COVID19 pandemic presents an opportunity to reimagine surgery and address factors that contribute to impaired wellness and Burnout. During this global pandemic, health systems and surgeons stepped out of their traditional roles and clinical comfort zones to care for patients and/or colleagues in need, and their efforts should be recognized. They adopted existing or new positive coping mechanisms to fight off social isolation and overcome challenges to their wellness. Surgeons are reporting more rest with longer hours of sleep and feeling more refreshed. Some found additional time for physical fitness, hobbies outside of work, and family. Time outside of work allows for introspection about family and occupational priorities, and whether or not previous levels of clinical productivity remain aligned with more balanced priorities. Technology, telepresence and time management are aspects of our modern daily life that contribute to the alignment of our priorities and may allow for sustained clinical, educational, and research productivity without sacrificing parts of our humanity that make us well. 


\section{Pursuing purpose, meaning, and fulfillment}

As one considers the issues surrounding physician wellness, it is apparent that many of our current efforts are on the defensive or mitigation front. While this is clearly important, flourishing as people involves active pursuit and engagement with energizing, meaningful and fulfilling activities, and not just avoiding pitfalls or managing stressors. In this regard, contemporary commentators note a default toward technical problem solving and systemic management strategies, as opposed to solutions based on character growth and the fulfillment of purpose [10,11]. Having an offensive rather than defensive or compensatory strategy for wellness is needed in the present context.

Studies on the theme of resilience in populations enduring high levels of stress identified practices that deter Burnout and promote thriving [12]. In studies of prisoners of war, landmine victims, and other groups suffering unusual pressures and trauma, Southwick and Charney highlight several practices that characterize thriving individuals [12]. These practices include optimism, adaptive responses to fear, developing a moral compass, dependence on faith, nurturing social support networks, using role models, maintaining physical and mental fitness, developing flexibility, and finding meaning and purpose in circumstances [12]. Other masterful philosophers and psychologists expressed similar themes regarding the issue of meaning and purpose. For example, Neitzsche said, "He [or she] who has a why can endure almost any how" [13]. Viktor Frankl commented on his experience counseling survivors of the Holocaust by saying, "Life is never made unbearable by circumstances, but only by lack of meaning and purpose" [14]. Analogous to the 'Band of Brothers' theme of Stephen Ambrose's writing and later film portrayal, it turns out that when seen as an opportunity for growth and contribution to the lives of others, even the most difficult circumstances can become the crucible for unusual human thriving, social connection, and contributions of enduring personal and social meaning and impact.

The relevance of these themes and their relationship to human motivation are summarized in Daniel Pink's book, "Drive" [15]. Pink points out that studies on productivity in domains that involve even a modicum of cognitive effort demonstrate that financial reward does not improve productivity and performance. What does matter is autonomy-the sense of ownership of the outcome and responsibility for the same; mastery - the hope that continued investment will lead to a product that is not only acceptable, but exquisite in its refinement and merit; and purpose or meaning - the awareness that what has been done is of enduring value as seen by the person responsible [15]. Clearly all these things are threatened in the current
Western medical paradigm that includes an emphasis on compliance with regulatory protocols, a disintegrated and volume-focused care delivery and reimbursement strategy, and commodification of the profession extending into areas of personal moral conviction and moral injury. The recent pandemic has demonstrated the bankruptcy of such a model not only literally, but in the failure to deliver population health, especially to the neediest elements of society. Accordingly, the present initiative focuses on reimagining or recapturing the meaning and purpose of the significant investment of time and energy involved in a surgical career. The existential implications of this initiative for surgeons, and the society that benefits from their presence and care, cannot be overstated.

While more complex and nuanced than a simple outline can capture, the following elements proved worthy of investment, offered in the spirit of one beggar telling another where they have found food. First, it is important to set and honor, meaning and fulfillment-based priorities in life. The illustration of 'getting the rocks right' captures this well, in which one tries to fit a few large rocks, a handful of small gravel, and a handful of sand into a glass jar. The only way to succeed at placing the rocks, gravel, and sand inside the jar is to place the largest objects first. Repeated decision making that does not reflect personal beliefs and priorities correlates with Burnout, which may be reflected as not spending at least $20 \%$ professional time on an area of passion [16], or as repeatedly favoring work in settings of work/home life temporal conflict [17]. More importantly for the present discussion, chronic time deployment can exclude important and sustaining elements of life from the overall orchestration and undermine purpose and meaning in work. Second, 'restore points' keep people whole; examples include spiritual disciplines such as meditation, prayer, and gratitude, physical exercise, enjoyment of nature or art in its various forms, or simply regular rest, such as observing sabbath or taking a sabbatical. Abandoning these 'restore points' undermines purposeful and strategic living, and may lend itself to a sprint, rather than the marathon of a surgical career. Third, given the nature of self-centered behavior and implosion of soul and society that accompanies it, focusing on investment in relational activities is important, both in work and non-work lives. Fourth, overcoming chronological snobbery, whereby people today compared to a prior age believe they possess a more mature understanding of what is meaningful and worthwhile in life because of the benefits enjoyed from science and technology. The recent 3-year decline in life expectancy related to so-called 'deaths of despair' (not witnessed in Western countries since World War I and the 1918 influenza pandemic that took 675,000 American lives) highlights the fact that despite our technologic and consumer amusements, people may know little of the meaning and value of life, which our science by definition cannot answer 
[18]. Seeking to give our attention not only to current flavors of thought but timeless themes by reading and giving attention to the wisdom of history is a helpful way to overcome chronological snobbery. A former President of the University of Southern California, Steven Sample, felt that great leaders shouldn't read anything not at least 400 years old, just to be sure the ideas they are imbibing are timelessly relevant [19].

People can also cultivate reflective habits and daily rhythms creating time to spend on what is important but not urgent, and therefore easily neglected [19]. These reflective habits and daily rhythms may be a common feature of genius and sustained high performance. Individuals can adopt a curricular view of life, especially with regard to character flaws, and recognize that much of character formation comes from facing those deficits and weaknesses [20]. People can build habits around these themes by becoming involved in communities both professionally and personally that enable and sustain such practices, and by learning from others with similar commitments [21]. Learning from like-minded individuals can be sustaining as the energy level of younger years fades into patterns sustainable only with collective strength and a broadened resource foundation. Relationships can minimize the error of thinking identity is only a product of career achievements and enable living that contributes to what will be said at our eulogy rather than what is on our resumes alone.

The pursuit of purpose, meaning, and fulfillment enfranchise patterns that make lives and careers sustainable, meaningful, and of enduring value. The prevention of Burnout and despair is as valuable as or more so, than strategies to mitigate and alleviate stressors.

\section{Leading with joy}

Where does joy come from? Does joy stem from professional pursuits alone, or from free time enjoying personal relationships? Does joy arise from financial security and the material goods or personal freedoms it can deliver? Surgeons may be vulnerable to a lack of joy, even Burnout when professional pursuits lose their meaning and purpose, a negative influence which can impact personal lives and relationships.

A paradigm shift in the profession of surgery would facilitate the rediscovery and reinvigoration of joy among surgeons and their family, friends, patients, and learners. Such a shift requires strong and dedicated leadership but also buy-in from those who operate within the paradigm. Burnout, evidenced by a lack of interest, loss of love for, and leaving the profession of surgery affects not only practicing surgeons but learners at various stages in their career. In truth, Burnout is a consequence of changes that deprive learners of the meaning and purpose they once found in their chosen profession. One study keyed in on potentially negative side effects from the current training paradigm. The SECOND TRIAL queried 7409 surgical residents from 262 training programs about discrimination and/or abuse in the workplace [22]. In all, 32\% of respondents reported some form of discrimination during their training period. Significantly more learners who experienced discrimination and/or abuse reported symptoms of Burnout (odds ratio 2.94; $95 \%$ CI 2.58 to 3.36) and suicidal thoughts (odds ratio 3.07; 95\% CI 2.25 to 4.19 ) compared to learners who did not experience discrimination [22]. In addition to this disturbing finding, learners today deal with increased patient workload, more documentation using EMR systems, decreased autonomy (cognitive and psychomotor), decreased camaraderie, decreased mentorship, and decreased team building events (social and academic). While anyone of these may be considered a challenge to overcome, it is no wonder that all combined may result in depersonalization, loss of meaning, and loss of purpose in work.

Healthcare is a universal connector so revitalizing joy in surgery must be multidisciplinary and community-based to respect and foster connectivity and teamwork. When a colleague or teammate shows personal or professional disconnect, intuitive and empathetic surgeon leaders must recognize these as symptoms suggestive of Burnout and take appropriate action. However, before taking action, the surgeon leader must understand the concept of mindfulness skills or mental coping strategies, which may be effective in mitigating stress in various work environments [23-26]. One mental coping strategy-achieving a restful mental state prior to performance-is known to improve performance of surgical residents [27]. Likewise, mental coping strategies can help improve technical skills particularly when delivered through organized curricula aimed at increasing resilience and self-reflection [26]. Moreover, surgeon leaders, as members of the healthcare team, should consider the lessons of improvement science to aid in developing a framework that would reinvigorate the pursuit of meaning and purpose by removing small, persistent impediments to joy. The Institute for Healthcare Improvement outlined those lessons and framework in The Joy of Work [28].

The team-based framework fashioned by the Institute for Healthcare Improvement uses a simple but effective four-step process to reintroduce joy to the work environment. The first step is to query colleagues and staff by sincerely and receptively asking, "What matters to you?" As a surgeon leader, it is important to empower other team leaders to ask the same question of their colleagues and staff, then close the loop of communication. As impediments to joy are identified, leaders should strategize solutions and sanction others to do the same. For best results, these impediments should be small, specific —a pebble in 
one's shoe. Oftentimes it may be best to divide the work among reliable and effective members of the team, thereby granting them both responsibility, authority and a level of autonomy for a given task. Honesty in such conversations opens doors to work as a team and develop mutual respect. The process itself can bring empowerment and joy regardless of the size of the pebble in the shoe. The third step involves a multidisciplinary team tasked with systematically and longitudinally removing impediments to joy. This step is critical to anticipate needs and mitigate risk of those impacted by any change. The fourth step is to use improvement science methodology for tracking, studying, and sharing all improvements to members of the healthcare community.

In order to meet the criteria set forth in the final step, and as a matter of honor and respect to all those who labor with passion, surgeon leaders and healthcare systems should create quality improvement projects aimed at removing impediments to joy. In fact, joy and positive patient outcomes are not coincidental. It is for surgeon leaders to remember that inspiring and empowering joy among a healthcare community may be a matter of finding the pebble and shaking it free from the shoe.

\section{Conclusion}

Physician and surgeon burnout has been a significant and progressively increasing issue over recent years. Burnout's rippling effects negatively influence patient care and health system effectiveness. The COVID-19 pandemic created a unique practice environment, providing surgeons with the opportunity to reflect and realign their personal and professional priorities toward wellness and away from Burnout. Initiatives such the rapid adoption of telehealth with minimal insurance-related hurdles, changed practice routines and delivery of care in ways which may prove to be beneficial. The increase in virtual meetings reduced travel and away time and may find a permanent place in our lives as we move forward.

Opportunities exist in the face of the COVID-19 pandemic to apply technology, consider meaning and purpose, and maintain quality patient care while improving self-care. While not a panacea, telehealth, rapidly accepted by patients, surgeons, health systems and payers, as well as online meeting platforms, allow for virtual yet personal interactions with patients and colleagues. Likewise, reflection on and identification of meaning and purpose in work and home life help surgeons strive for wellness. Surgeon wellness, finding joy at work and self-care, must be considered important factors in the future of all healthcare delivery systems. This need has been strongly reaffirmed by the COVID-19 pandemic.
Acknowledgements SAGES REIMAGINING THE PRACTICE OF SURGERY TASK FORCE: Chair: Liane S Feldman; Co-Chairs: James G. Bittner and Edward Auyang. Members: Gina Adrales, Adnan Alseidi, Horacio Asbun - President, Jonathan Dort , Denise Gee, David Geller, Rana Higgins, Renee Hilton, Farah Husain, Hope Jackson, Rohan Jeyarajah, Debby Keller, Lawrence Lee, Amin Madani, John Mellinger, Phil Omotosho, Eric Pauli, Kevin Reavis, Dave Renton, John Romanelli, Barry Salky, Pay Sylla, Shawn Tsuda, Linda Zhang.

\section{Compliance with ethical standards}

Disclosures Dr Romanelli reports personal fees from New View Surgical, outside the submitted work. Dr Gee reports ownership interest in New View Surgical, personal fees from Medtronic, personal fees from Coviden, piuotside the submitted work. Dr Bittner reports personal fees from Intuitive Surgical Inc., grants and personal fees from BD, Inc., personal fees from CMR Surgical LTD, personal fees from Cook Biotech Inc., outside the submitted work. Drs Auyang, AlSeidi, Feldman, Asbun and Mellinger have no conflicts or interest or financial ties to disclose.

\section{References}

1. Hartzband P (2020) Groopman J (2020) Physician burnout, interrupted. NEJM 382:2485-2487

2. Senturk JC, Melnitchouk N (2019) Surgeon burnout: defining, identifying and addressing the new reality. Clin Colon Rectal Surg 32:407-414

3. Maslach C, Schaufeli WB, Maslach C, Marek T (eds) (1993) Professional burnout: recent developments in theory and research. Taylor \& Francis, Washington DC

4. Author (2020) https://www.medscape.com/slideshow/2020-lifes tyle-burnout-6012460. Accessed 7 February 2020.

5. Gawande A (2018) Why doctors hate their computers. https:// www.newyorker.com/magazine/2018/11/12/why-doctors-hatetheir-computers. Accessed 28 May 2020.

6. Shanafelt TD, Balch CM, Bechamps GJ et al (2009) Burnout and career satisfaction among American surgeons. Ann Surg 250:463-471

7. Dyrbye LN, Burke SE, Hardeman RR et al (2018) JAMA 320:1114-1130

8. Elmore LC, Jeffe DB, Jin L, Awad MM, Turnbull IR (2016) National survey of Burnout among US general surgery residents. J Am Coll Surg 223:440-451

9. Dort J, Romanelli J, Choudry N et al (2020) SAGES primer for taking care of yourself during and after the COVID-19 crisis. Surg Endosc 34:2856-2862

10. Einstein A (1941) The common language of science. Broadcast for Science Conference; 28 September.

11. Eliot TS (1963) Choruses from the rock. Collected Poems. Houghton Mifflin Harcourt, New York, pp 1909-1962

12. Southwick SM, Charney DS (2012) Resilience: the science of mastering life's greatest challenges. Cambridge University Press, Cambridge

13. Nietzsche F (1896) The twilight of the idols. Transl, Common T. H Henry and Company, London

14. Frankl VE (1984) Man's search for meaning: an introduction to logotherapy. Simon \& Schuster, New York

15. Pink DH (2009) Drive: the surprising truth about what motivates us. Riverhead Books, New York

16. Shanafelt TD, West CP, Sloan JA et al (2009) Career fit and burnout among academic faculty. Arch Intern Med 169:990-995 
17. Dyrbye LN, Shanafelt TD, Balch CM et al (2011) Relationship between work-home conflicts and Burnout among American surgeons: a comparison by sex. Arch Surg 146:211-217

18. Brooks D (2019) The second mountain: the quest for a moral life. Random House, New York

19. Sample SB (2003) The contrarian's guide to leadership. JosseyBass, San Francisco

20. Brooks D (2016) The road to character. Random House, New York

21. Duhigg C (2012) The power of habit: why we do what we do in life and business. Random House, New York

22. Hu YY, Ellis RJ, Hewitt DB et al (2019) Discrimination, abuse, harassment, and Burnout in surgical residency training. N Engl J Med 381:1741-1752

23. Anton NE, Beane J, Yurco A et al (2018) Mental skills training effectively minimizes operative performance deterioration under stressful conditions: results of a randomized controlled study. Am J Surg 215:214-221

24. Anton NE, Howley LD, Pimentel M et al (2016) Effectiveness of a mental skills curriculum to reduce novices' stress. J Surg Res 206:199-205
25. Stefanidis D, Anton NE, McRary G et al (2017) Implementation results of a novel comprehensive mental skills curriculum during simulator training. Am J Surg 21:353-361

26. Anton NE, Mulji N, Howley LD et al (2017) Effects of a novel mental skills curriculum on surgical novices' attention. J Surg Res 219:86-91

27. Stefanidis D, Anton NE, Howley LD et al (2017) Effectiveness of a comprehensive mental skills curriculum in enhancing surgical performance: results of a randomized controlled trial. Am J Surg 213:318-324

28. Perlo J, Balik B, Swensen S, Kabcenell A, Landsman J, Feeley D (2017) IHI Framework for Improving Joy in Work. Institute for Healthcare Improvement, Cambridge, MA

Publisher's Note Springer Nature remains neutral with regard to jurisdictional claims in published maps and institutional affiliations. 